\title{
Statistical bounds on the dynamical production of entanglement
}

\author{
Rômulo F. Abreu* and Raúl O. Vallejost \\ Centro Brasileiro de Pesquisas Físicas (CBPF), \\ Rua Dr. Xavier Sigaud 150, 22290-180 Rio de Janeiro, Brazil
}

(Dated: April 25, 2017)

\begin{abstract}
We present a random-matrix analysis of the entangling power of a unitary operator as a function of the number of times it is iterated. We consider unitaries belonging to the circular ensembles of random matrices (CUE or COE) applied to random (real or complex) non-entangled states. We verify numerically that the average entangling power is a monotonic decreasing function of time. The same behavior is observed for the "operator entanglement" -an alternative measure of the entangling strength of a unitary. On the analytical side we calculate the CUE operator entanglement and asymptotic values for the entangling power. We also provide a theoretical explanation of the time dependence in the CUE cases.

PACS numbers: 03.65.Ud, 03.65.Yz, 05.45.Mt
\end{abstract}

*Electronic address: romulof@cbpf.br

$\dagger$ Electronic address: vallejos@cbpf.br; URL: http://www.cbpf.br/ vallejos 


\section{INTRODUCTION}

In the last years many studies were devoted to the determination of entanglement growth laws for bipartite pure states evolving from product states under globally unitary dynamics, either with continuous [1, 2, 3, 4, 5, 6, 7, 8] or discrete time [9, 10, 11, 12, 13, 14, 15, 16, 17, 18]. For not too small systems, and weak couplings between subsystems, the general qualitative picture is that of entanglement (subsystem entropy) growing smoothly from zero, possibly in a nonmonotonic way, until arriving at an asymptotic regime characterized by small oscillations around an equilibrium value. However, when we come to the quantitative level a rich phenomenology is discovered [2, 3, 4, 5, 6, 7, 8, 11, 12, 13, 14, 15, 16, 17, 18]. Besides chaos or regularity at the classical level, the choice of parameters like subsystem dimensions, coupling strength, initial state, time window, etc., play also important roles in determining the law of growth of entropy [19].

In this paper we concentrate on the regime of very long times, i.e., after the system has relaxed to a equilibrium state. More precisely, we are interested in the average value of the asymptotic entropy over a suitable distribution of initially nonentangled states. This defines the asymptotic entangling power [20] of the unitary dynamics.

If the classical dynamics is chaotic in the full phase space, then, according to the BohigasGiannoni-Schmit conjecture [21, 22], one should expect that Random Matrix Theory will succeed in describing the statistical features of the long time dynamics, in particular, the distribution of asymptotic entropies. However, there is a much simpler statistical approach, based on the assumption that a typical initial state submitted to a "chaotic" dynamics must eventually evolve into a random state, uniformly distributed on the sphere, as far as its average properties are concerned. This hypothesis was tested in several finite dimensional quantum maps, with a satisfactory quantitative agreement between theory and simulation [12, 14, 15, 23, 24$]$.

The purpose of this paper is to compare the predictions of Random Matrix Theory and the alluded "Random State Theory" for the average asymptotic entanglement generated by a globally unitary map. In Random Matrix Theory the dynamics is explicitly introduced in the model: an asymptotic state is generated by the repeated application of a random unitary map to a nonentangled initial state [2]. We show that the ensemble of states generated in this way does not coincide in general with a uniform distribution on the sphere. 
Our results are more conveniently stated in a language of operators: the entangling power [20] of $U^{n}$, where $U$ is a random unitary, decreases (in average) with increasing the discrete time $n$. The statement continues to be true if one substitutes "entangling power" by "operator entanglement" [25, 26, 27], another useful measure of the entangling abilities of a unitary (verified numerically, section (III).

The following section (III) contains the definitions and the exact setting of the problem. Sections III and IV present our numerical and analytical results, respectively. A brief discussion of the results is left to section $\mathrm{V}$.

\section{DEFINITIONS AND SETTING}

We restrict our analysis to the case of bipartite entanglement of pure states in finite dimensional Hilbert spaces. As a measure of entanglement, we use the subsystem linear entropy.

Consider a full system divided into two subsystems, $A$ and $B$. The dimension of the full Hilbert space $\mathcal{H}$ is $d=d_{A} d_{B}$, with $d_{A}$ and $d_{B}$ the subsystem dimensions. Let $|\psi\rangle=$ $\left|\psi_{A}\right\rangle \otimes\left|\psi_{B}\right\rangle$ be a pure separable state of the full system, corresponding to the density matrix $\rho=|\psi\rangle\langle\psi|$. In general, after $n$ applications of $U, n \geq 1$, the new density matrix $\rho^{(n)}=U^{n} \rho U^{n \dagger}$ will not correspond to a separable state any more, due to the increasing entanglement between the subsystems. This will manifest itself in a growth of the linear entropy of the reduced density matrices

$$
S_{L}^{(n)}(|\psi\rangle) \equiv 1-\operatorname{tr}\left[\rho_{A}^{(n)}\right]^{2}=1-\operatorname{tr}\left[\rho_{B}^{(n)}\right]^{2}
$$

where $\rho_{A}^{(n)}=\operatorname{tr}_{B} \rho^{(n)}$ and $\rho_{B}^{(n)}=\operatorname{tr}_{A} \rho^{(n)}[28]$. For long times, and typical $U$ and $|\psi\rangle$, the system comes into an equilibrium regime, where the linear entropy shows small fluctuations around a stationary average (see, e.g., [12, 14, 15, 16, 23]), given by

$$
S_{L}^{\infty}(|\psi\rangle) \equiv \lim _{N \rightarrow \infty} \frac{1}{N} \sum_{n=1}^{N} S_{L}^{(n)}(|\psi\rangle) .
$$

By doing an additional average on initial product states one arrives at the asymptotic entangling power of $U$ :

$$
e p^{\infty}(U) \equiv\left\langle S_{L}^{\infty}(|\psi\rangle)\right\rangle_{|\psi\rangle=\left|\psi_{A}\right\rangle \otimes\left|\psi_{B}\right\rangle}
$$


It is also useful to consider a time-dependent entangling power, i.e., the initial-state average of Eq. (1):

$$
e p^{(n)}(U) \equiv\left\langle S_{L}^{(n)}(|\psi\rangle)\right\rangle_{|\psi\rangle=\left|\psi_{A}\right\rangle \otimes\left|\psi_{B}\right\rangle} .
$$

For $n=1$ this is just the entangling power of $U$.

Concerning the average over product states, we take $\left|\psi_{A}\right\rangle$ and $\left|\psi_{B}\right\rangle$ to be independent random vectors, both of them either real or complex, uniformly drawn from the corresponding sphere [29, 30]. In other words, the components of $\left|\psi_{A}\right\rangle$ and $\left|\psi_{B}\right\rangle$ are distributed like the columns of a matrix belonging either to the orthogonal group (real case) or unitary group (complex case) (Haar measure is assumed in both cases). There are two reasons for these choices. (i) They are perhaps the simplest nontrivial cases both from a conceptual point of view [29] and from the perspective of analytical calculations. (ii) They will allow us to make contact with closely related literature (e.g., Refs. [2, 12, 14, 20]).

The problem is how to estimate $e p^{\infty}(U)$ for a typical unitary $U$. For "typical unitary" we mean an operator describable (in a statistical sense) by any of the Circular Ensembles of Random Matrix Theory (RMT) [31] . Accordingly we shall consider that $U$ belongs either to the Circular Unitary Ensemble (CUE, unitary group with Haar measure) or to the Circular Orthogonal Ensemble (COE), the latter being the appropriate choice for unitaries displaying time reversal symmetry [22]. This leaves us with four cases to analyze: CUE/COE unitaries acting on random complex/real states.

In order to check that our results are not exclusive of the measure chosen for quantifying entangling strength [32], in addition to "entangling power" we also studied the alternative measure called operator entanglement [25] (also known as Schmidt strength [27]), constructed as follows. A bipartite Hilbert space induces a bipartite structure in the space of its linear operators, which, equipped with the Hilbert-Schmidt product becomes a bipartite Hilbert space itself. Then, operators can be treated as usual vectors, and standard measures for entanglement of states can be translated to operators [20, 26, 30, 33]. For instance, the linear entropy of the unitary $U$ reads [26]

$$
S_{L}(U)=1-\frac{1}{d_{A}^{2} d_{B}^{2}} \sum_{k_{1}, k_{2}, l_{1}, l_{2}=1}^{d_{A}} \sum_{i_{1}, i_{2}, j_{1}, j_{2}=1}^{d_{B}} U_{k_{1} i_{1}, k_{2} i_{2}} U_{l_{1} j_{1}, l_{2} j_{2}} U_{l_{1} i_{1}, l_{2} i_{2}}^{*} U_{k_{1} j_{1}, k_{2} j_{2}}^{*},
$$

where the matrix elements of $U$ are related to a product basis, i.e.,

$$
U_{k_{1} i_{1}, k_{2} i_{2}}={ }_{A}\left\langle\left. k_{1}\right|_{B}\left\langle i_{1}|U| k_{2}\right\rangle_{A} \mid i_{2}\right\rangle_{B}
$$


Of course, by substituting $U$ by $U^{n}$ in the equations above we obtain the operator entanglement as a function of time.

\section{NUMERICAL RESULTS}

We start with a numerical study, emphasizing the most interesting features, but postponing a deeper analysis until section IV.

The main ingredients of our simulations are random states (real or complex) and random matrices (CUE or COE). They were generated using the same methods as in Ref. [24]. Random states are evolved by applying $n$ times a quantum random map. Then, the entropy of the final state is calculated and averaged over maps and (if necessary) over states.

Figure 1 shows the entangling power of a unitary as a function of time, averaged over CUE $[(a)]$ or COE $[(b),(c)]$. The cases $(a, b)$ and $(c)$ correspond, respectively, to complex or real initial states. In cases (a) and (c), due to invariance considerations, the average over states is redundant and it suffices to consider a single state. For similar reasons, the cases $n=1$ represent the average linear entropy of standard bipartite pure states, either complex $[(\mathrm{a})]$ or real $[(\mathrm{b}),(\mathrm{c})]$ (see section [V]).

In the three cases we observe that average entanglement is a decreasing function of the number of iterations. This is the opposite to what is observed in weakly coupled maps, i.e., entropy increasing from a zero initial value. However, we remark that our purpose is to model the equilibrium itself, not the initial phase of relaxation to equilibrium -this would require an explicit modeling of the weak coupling, as in Ref. [2]. The cases $n=1$ and $n \rightarrow \infty$ correspond, respectively, to the predictions of "Random State Theory" and Random Matrix Theory. Even though these extreme cases are our main concern, we also analyze the regime of intermediate times because it contains valuable information, e.g., about characteristic times for the transition between the extremes.

Evidently the characteristic time for saturation is the Heisenberg time $n_{H} \equiv d$ (in our simulations $d=20)$. In the CUE case the saturation happens abruptly at $n=n_{H}$. We also verified that the operator entanglement behaves in a similar way by plotting the average linear entropy of $U^{n}$ as a function of $n$ (see Fig. 2).

Both figures exhibit a very curious characteristic: the asymptotic value for CUE maps coincides, within numerical precision, with the $n=1$ value for COE. We shall see in the 


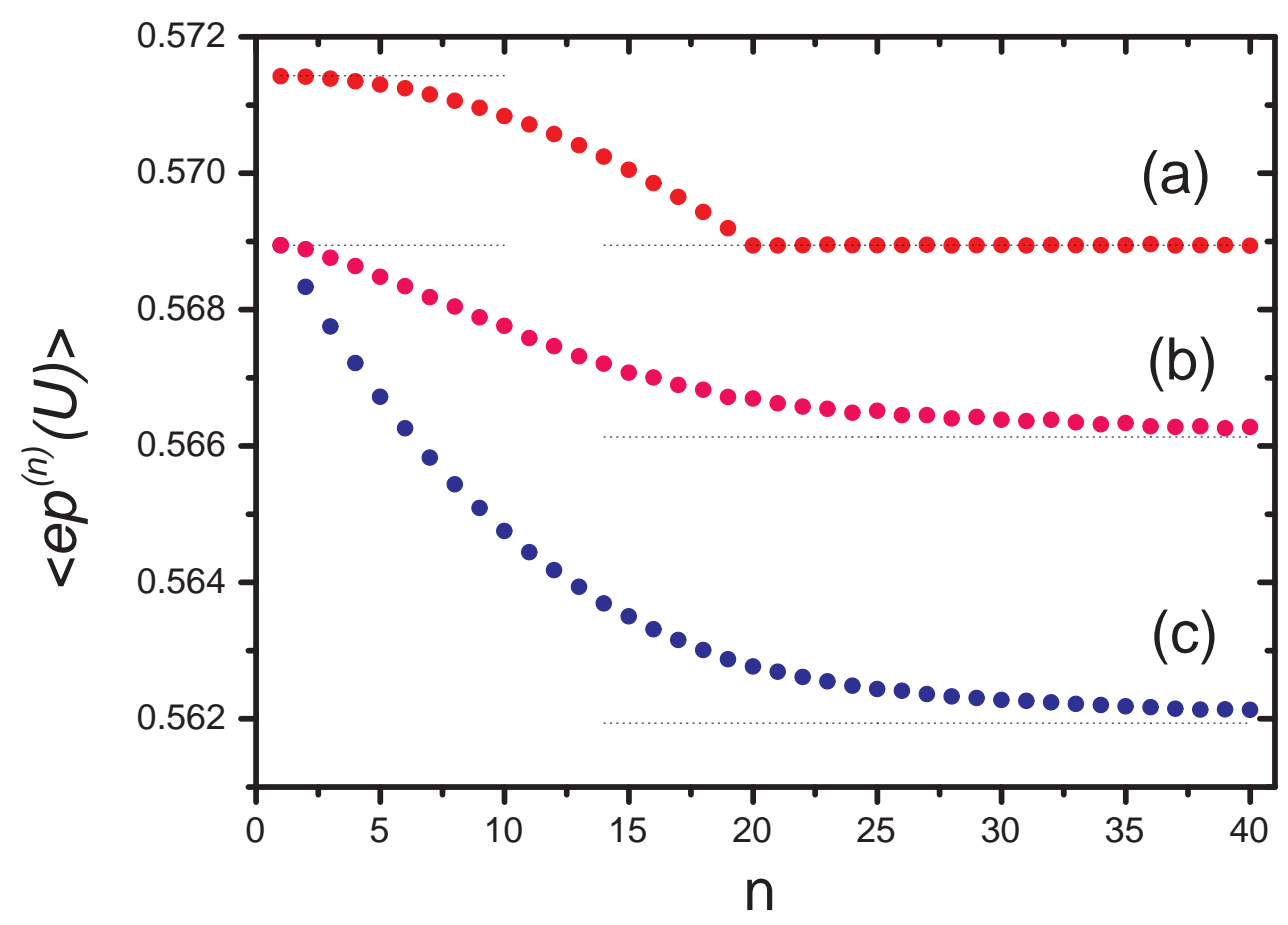

FIG. 1: (a) A fixed initial separable state was chosen arbitrarily and then propagated by $U^{n}$, with $U$ belonging to CUE. Shown is the linear entropy of the evolved states averaged over $10^{8}$ CUE matrices. (b) $10^{5} \mathrm{COE}$ matrices were used to propagate an ensemble of $10^{3}$ random, complex, separable initial states. Shown is the linear entropy averaged over COE matrices and states. (c) Idem (a) but for $10^{8} \mathrm{COE}$ matrices and one real state. In all cases subsystem dimensions are $d_{A}=4$ and $d_{B}=5$. Statistical error bars are smaller than symbol diameter. Dotted lines correspond to analytical predictions, see section IV.

next section that, in the case of the entangling power (Fig. 1), such a coincidence is indeed exact, for all subsystem dimensions $d_{A}$ and $d_{B}$.

\section{ANALYTICAL RESULTS}

The purpose of this section is to explain analytically some of the features present in Fig. (11) and Fig. (2). In some cases we shall be able to understand the global appearance of the entangling measures as functions of time, and derive quantitative expressions for some 


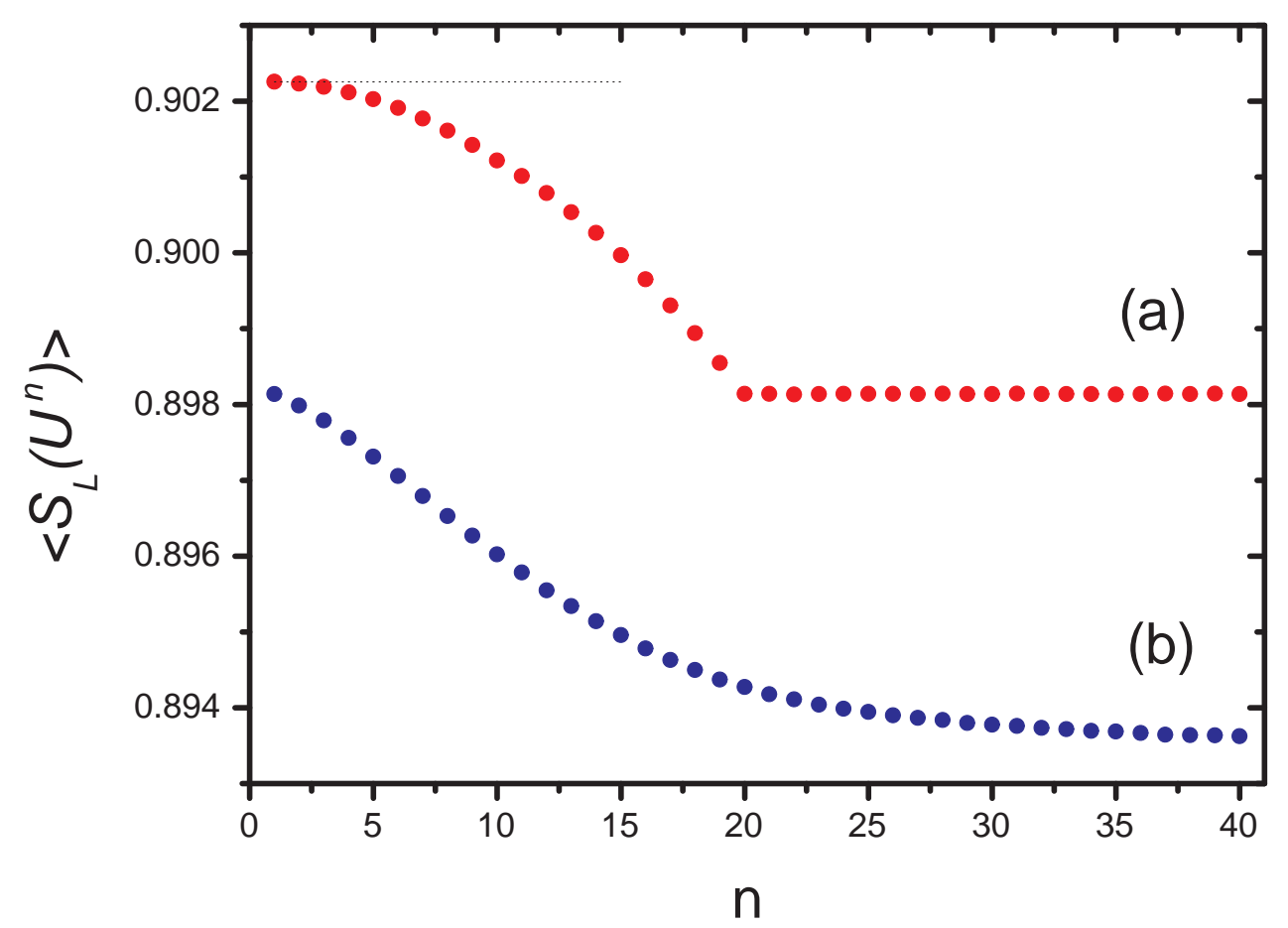

FIG. 2: Shown is the linear entropy of the random operator $U^{n}$ averaged over $10^{7}$ realizations. (a) $U$ belongs to CUE. (b) $U$ belongs to COE. In both cases subsystem dimensions are $d_{A}=4$ and $d_{B}=5$. Statistical error bars are smaller than symbol diameter. Dotted line correspond to the analytical prediction, see section IV.

limiting values (indicated with dotted lines in Figs. 11 and 22).

The values for the entangling power at $n=1$ can already be found in the literature

$$
\begin{aligned}
e p^{(1)}(U)_{(a)} & =\frac{d-\left(d_{A}+d_{B}\right)+1}{d+1}, \\
e p^{(1)}(U)_{(b),(c)} & =\frac{d^{3}-\left(d_{A}+d_{B}-4\right) d^{2}-\left[3\left(d_{A}+d_{B}\right)-1\right] d+2\left(d_{A}+d_{B}-1\right)}{d(d+1)(d+3)} .
\end{aligned}
$$

[Subindices (a), (b), (c) refer to each one of the cases depicted in Fig. 1], The first equality above corresponds to the well known entropy of random complex states [30]. The second result can be found in Ref. [2].

Concerning operator entanglement, the case $n=1$ was calculated by Zanardi [25] for two qudits, i.e., $d_{A}=d_{B}$. Using techniques to be described below, we obtained the CUE average 
of Eq. (5):

$$
\left\langle S_{L}(U)\right\rangle_{\mathrm{CUE}}=\frac{d^{2}-\left(d_{A}^{2}+d_{B}^{2}\right)+1}{d^{2}-1},
$$

thus extending Zanardi's result to arbitrary subsystem dimensions. Inserting $d_{A}=4$ and $d_{B}=5$ in this formula we obtain the value indicated with dotted lines in Fig. 2 .

\section{A. Global features}

All the functions depicted in Figs. 1 1 and 2 share the property of decreasing in a monotonic way and coming to saturation around the Heisenberg time. Or, equivalently, one can say that the purity (one minus linear entropy) grows and then saturates. The most surprising case is CUE, because of the abrupt saturation at $n=n_{H}$. This behavior is similar to that of the form factor of the Circular Ensembles [22], defined as

$$
\left\langle\left|\operatorname{tr} U^{n}\right|^{2}\right\rangle \equiv\left\langle\left|t_{n}\right|^{2}\right\rangle=\sum_{\alpha, \beta=1}^{d}\left\langle e^{i n\left(\phi_{\alpha}-\phi_{\beta}\right)}\right\rangle,
$$

where the average runs over CUE or COE. For CUE the form factor is piecewise linear:

$$
\left\langle\left|t_{n}\right|^{2}\right\rangle_{\mathrm{CUE}}=\left\{\begin{array}{lll}
n & \text { if } & 1 \leq n \leq d \\
d & \text { if } & n \geq d .
\end{array},\right.
$$

The explanation for this behavior is as follows. The form factor is a function only of the eigenvalues of $U^{n}$. If $n=1$ one has the well known random matrix spectrum which shows strong correlations, e.g., level repulsion. For $n>1$ the spectrum has been stretched and folded $n$ times on the unit circle, and, when $n \gtrsim d$ the spectrum is almost completely uncorrelated [22]. Evidently, the same mechanism is responsible for the saturation of the entangling measures.

The similarity between purity and form factor was already noted by Gorin and Seligman [2], who considered a continuous time Hamiltonian analogue of the COE case in Fig. 1(c). Now we show that such a connection can be established rigorously for CUE maps. Consider either the entangling power or the operator entanglement, Eqs. 4 and [5, respectively; insert the spectral decomposition for the corresponding unitaries. We recall that eigenvectors and eigenvalues are statistically independent in the circular ensembles. In all cases the result can be written as follows:

$$
S(n)=1-\sum_{\alpha, \beta, \delta, \gamma=1}^{d} C_{\alpha \beta \delta \gamma}\left\langle e^{i n\left(\phi_{\alpha}+\phi_{\beta}-\phi_{\delta}-\phi_{\gamma}\right)}\right\rangle .
$$


On the left, $S(n)$ represents any of the average entropies considered. The coefficients $C_{\alpha \beta \delta \gamma}$ contain the average over eigenvectors and (where applicable) initial states. The time dependence comes from the average over four eigenphases $\phi$. Due to invariance properties of CUE and COE, the averages above do not depend on the particular values of the indices $\alpha, \beta, \delta, \gamma$, but only on their being all different, all equal, equal in pairs, etc. Thus, one is left with the problem of evaluating a few nontrivial averages [2]:

$$
\begin{aligned}
& \left\langle\exp \left[i n\left(\phi_{\alpha}+\phi_{\beta}-\phi_{\delta}-\phi_{\gamma}\right)\right]\right\rangle, \\
& \left\langle\exp \left[i n\left(2 \phi_{\alpha}-\phi_{\delta}-\phi_{\gamma}\right)\right]\right\rangle, \\
& \left\langle\exp \left[2 i n\left(\phi_{\alpha}-\phi_{\delta}\right)\right]\right\rangle, \\
& \left\langle\exp \left[i n\left(\phi_{\alpha}-\phi_{\delta}\right)\right]\right\rangle .
\end{aligned}
$$

For CUE, we can show that all these four averages can be expressed in terms of the basic form factors [34]

$$
\left\langle\left|t_{n}\right|^{2}\right\rangle^{2},\left\langle\left|t_{2 n}\right|^{2}\right\rangle,\left\langle\left|t_{n}\right|^{2}\right\rangle
$$

[this is immediate for averages (15) and (16)]. The information we have gathered is enough for concluding that in CUE cases one must have

$$
S(n)=c_{1}+c_{2}\left\langle\left|t_{n}\right|^{2}\right\rangle^{2}+c_{3}\left\langle\left|t_{2 n}\right|^{2}\right\rangle+c_{4}\left\langle\left|t_{n}\right|^{2}\right\rangle
$$

where $c_{k}$ are certain time-independent coefficients.

This result is not unexpected, as the same three basic functions above also appear in the CUE average of $\left|t_{n}\right|^{4}$, calculated by Haake some years ago [22],

$$
\left\langle\left|t_{n}\right|^{4}\right\rangle=2\left\langle\left|t_{n}\right|^{2}\right\rangle^{2}+\left\langle\left|t_{2 n}\right|^{2}\right\rangle-2\left\langle\left|t_{n}\right|^{2}\right\rangle
$$

and $\left\langle\left|t_{n}\right|^{4}\right\rangle$ is structurally very similar to the entangling measures we are considering:

$$
\left\langle\left|t_{n}\right|^{4}\right\rangle=\sum_{\alpha, \beta, \delta, \gamma=1}^{d}\left\langle e^{i n\left(\phi_{\alpha}+\phi_{\beta}-\phi_{\delta}-\phi_{\gamma}\right)}\right\rangle .
$$

Whatever the exact values of $c_{k}$ in Eq. (18), the preceding analysis proves that for CUE both entangling power and operator entanglement decay quadratically and then saturate abruptly. (Strictly speaking the decay is piecewise quadratic; however this effect is not perceptible in our figures, neither is it in a plot of $\left\langle\left|t_{n}\right|^{4}\right\rangle$ versus $n[34]$.)

The possible relationship between the form factor and the entangling measures in the COE cases remains a conjecture (Gorin-Seligman's); the required calculations are rather more difficult and will not be attempted here. 


\section{B. Asymptotic values}

As in the preceding subsection, the starting point for the calculations of asymptotic values is the general expression (12). In the case of the entangling power the coefficients $C_{\alpha \beta \delta \gamma}$ are the result of a double average over eigenvectors $\left|e_{\mu}\right\rangle$ and initial states $|\psi\rangle$ [15],

$$
C_{\alpha \beta \delta \gamma}=\left\langle\left\langle\left\langle e_{\alpha} \mid \psi\right\rangle\left\langle\psi \mid e_{\delta}\right\rangle\left\langle e_{\beta} \mid \psi\right\rangle\left\langle\psi \mid e_{\gamma}\right\rangle \operatorname{tr}_{A}\left[\operatorname{tr}_{B}\left(\left|e_{\alpha}\right\rangle\left\langle e_{\delta}\right|\right) \operatorname{tr}_{B}\left(\left|e_{\beta}\right\rangle\left\langle e_{\gamma}\right|\right)\right]\right\rangle\right\rangle
$$

The calculation of the asymptotic entangling power requires the time average (2) which washes out the eigenvalue dependence but enforces the pairing of indices: $\alpha=\delta$ and $\beta=\gamma$, or $\alpha=\gamma$ and $\beta=\delta$. Thus, one arrives at [15, 24]

$e p^{\infty}(U)=1-\left\langle\left\langle\sum_{\alpha}\left|\left\langle e_{\alpha} \mid \psi\right\rangle\right|^{4} \operatorname{tr}_{A}\left(\rho_{A}^{\alpha}\right)^{2}-\sum_{\alpha \neq j}\left|\left\langle e_{\alpha} \mid \psi\right\rangle\right|^{2}\left|\left\langle e_{\beta} \mid \psi\right\rangle\right|^{2}\left[\operatorname{tr}_{A}\left(\rho_{A}^{\alpha} \rho_{A}^{\beta}\right)+\operatorname{tr}_{B}\left(\rho_{B}^{\alpha} \rho_{B}^{\beta}\right)\right]\right\rangle\right\rangle$,

where $\rho_{A}^{\alpha}$ and $\rho_{B}^{\alpha}$ stand for the reduced density matrices of the eigenvector $\left|e_{\alpha}\right\rangle$ :

$$
\begin{aligned}
& \rho_{A}^{\alpha}=\operatorname{tr}_{B}\left|e_{\alpha}\right\rangle\left\langle e_{\alpha}\right|, \\
& \rho_{B}^{\alpha}=\operatorname{tr}_{A}\left|e_{\alpha}\right\rangle\left\langle e_{\alpha}\right| .
\end{aligned}
$$

In cases (a) and (c) of Fig. 1 the average over initial states is redundant. It suffices to consider just one fixed initial product state. This is due to the invariance of the Haar measure with respect to left/right group actions, either for the unitary [(a)] or the orthogonal [(c)] group, combined with the fact that local operations do not change the entropy [2] (recall that the eigenvectors of CUE and COE are Haar distributed in the unitary and orthogonal groups, respectively). So, in cases (a) and (c) we fix the initial state, e.g., $|\psi\rangle=|1\rangle_{A} \otimes|1\rangle_{B}$. In case (b) we must average $|\psi\rangle_{A}$ and $|\psi\rangle_{B}$ over their respective spheres. In the unitary case (a) one has:

$$
\begin{aligned}
e p^{\infty}(U)_{(\mathrm{a})=1-\langle} & \sum_{\alpha} \sum_{r, r^{\prime}, s, s^{\prime}}\left|U_{11, \alpha}\right|^{4} U_{r s, \alpha} U_{r^{\prime} s, \alpha}^{*} U_{r^{\prime} s^{\prime}, \alpha} U_{r s^{\prime}, \alpha}^{*}- \\
& \sum_{\alpha \neq \beta} \sum_{r, r^{\prime}, s, s^{\prime}}\left|U_{11, \alpha}\right|^{2}\left|U_{11, \beta}\right|^{2} U_{r s, \alpha} U_{r^{\prime} s, \alpha}^{*} U_{r^{\prime} s^{\prime}, \beta} U_{r s^{\prime}, \beta}^{*}- \\
& \left.\sum_{\alpha \neq \beta} \sum_{r, r^{\prime}, s, s^{\prime}}\left|U_{11, \alpha}\right|^{2}\left|U_{11, \beta}\right|^{2} U_{r s, \alpha} U_{r s^{\prime}, \alpha}^{*} U_{r^{\prime} s^{\prime}, \beta} U_{r^{\prime} s, \beta}^{*}\right\rangle .
\end{aligned}
$$

The expression for the orthogonal case (c) is identical to the preceding one but for $U$ a real unitary matrix. The remaining case, (b), will be exhibited in Ref. [34]. 
In all cases, the last step is a group average of products of eight matrix elements (not always different) belonging to one or two columns, i.e., "one- and two-vector averages of monomials of order eight". For such averages we used the powerful diagrammatic method devised by Aubert and Lam for the unitary group [35] and adapted by Braun to the orthogonal case [36]. The method is based solely on the unitarity/orthogonality constraint and the invariance of the Haar measure under the group actions. It provides explicit expressions for some integrals and recurrence relations for others. As the calculations are lengthy but otherwise not illuminating, we skip intermediate steps [34] and jump to the final results:

$$
\begin{aligned}
e p^{\infty}(U)_{(\mathrm{a})} & =e p^{(1)}(U)_{(b),(c)}, \\
e p^{\infty}(U)_{(\mathrm{c})} & =\frac{d^{4}-\left(d_{A}+d_{B}-13\right) d^{3}-\left[12\left(d_{A}+d_{B}\right)-47\right] d^{2}-35\left(d_{A}+d_{B}-1\right) d}{(d+1)(d+2)(d+4)(d+6)} .
\end{aligned}
$$

The first line says that the asymptotic average entropy in the unitary ensemble coincides with the $n=1$ value for COE [see Eq. (8)], for all dimensions $d_{A}$ and $d_{B}$. This confirms the suspicion caused by examining the data in Fig. 1. However, we have not been able to go beyond the mere analytical verification of the conjecture. The deep reasons for such a coincidence -if any- remain a mystery.

The second expression agrees with Gorin-Seligman's calculation [2], who used a different method for averaging monomials over the orthogonal group [37].

For the case (b) we obtained

$$
e p^{\infty}(U)_{(\mathrm{b})}=\frac{X}{Y},
$$

with

$$
\begin{aligned}
X= & d^{5}+12 d^{4}-\left(d_{A}^{2}+d_{B}^{2}-41\right) d^{3}- \\
& {\left[12\left(d_{A}^{2}+d_{B}^{2}\right)+2\left(d_{A}+d_{B}\right)-30\right] d^{2}-} \\
& {\left[38\left(d_{A}^{2}+d_{B}^{2}\right)+18\right] d-16\left(d_{A}^{2}+d_{B}^{2}\right)+56\left(d_{A}+d_{B}\right)-40, } \\
Y= & \left(d_{A}+1\right)\left(d_{B}+1\right)(d+1)(d+2)(d+4)(d+6) .
\end{aligned}
$$

One of the advantages of having explicit analytical expressions is that we can now quantify the differences between $n=1$ and $n \rightarrow \infty$. For CUE this represents the difference between the predictions of theories based either on random states or on random dynamics. For instance, let us consider the scaling with system size, taking for definiteness $d_{A}=2$ (which 
can be thought of as a definition of entangling power according to the multiqubit MeyerWallach measure [38]). Then, for large $d_{B}$ one has

$$
\begin{aligned}
& e p^{(1)}(U)_{(\mathrm{a})}-e p^{\infty}(U)_{(\mathrm{a})}=\frac{1}{4 d_{B}^{2}}+\ldots, \\
& e p^{(1)}(U)_{(\mathrm{b})}-e p^{\infty}(U)_{(\mathrm{b})}=\frac{1}{3 d_{B}^{2}}+\ldots, \\
& e p^{(1)}(U)_{(\mathrm{c})}-e p^{\infty}(U)_{(\mathrm{c})}=\frac{7}{8 d_{B}^{2}}+\ldots .
\end{aligned}
$$

So, the differences are always of second order in the system size.

\section{CONCLUSIONS}

An initially nonentangled state evolving under a globally chaotic dynamics displays asymptotically features of randomness. This can be modeled by assuming that the state becomes a completely random state, i.e., uniformly distributed on the sphere. Alternatively, one can assume that randomness lies in the dynamics, and find out which is the ensemble of final states obtained in this way. We showed that both ensembles are different, i.e., the dynamics, even if chaotic, does not generate "canonical" random states. When one includes in the model the information that states are generated dynamically, the ensemble-average entropy decreases due to additional correlations among the state components. This shows up as the difference $n=1$ vs. $n \rightarrow \infty$. The effect is relatively small, i.e., second-order in system size, but it can be clearly detected in our figures, and might be important for small systems.

A curious byproduct of our studies is the conclusion that the asymptotic entangling measures for CUE operators coincide with the respective $n=1 \mathrm{COE}$ cases. Thus, the effect of explicitly including the dynamics in the statistical modeling is equivalent to imposing a time reversal symmetry.

Our results contain also a warning against excessively strong interpretations of the Bohigas-Giannoni-Schmit conjecture, which associates classical chaos with quantum randomness. Naively, one may be led to believe that "more chaos always leads to more entanglement". However, if $U$ is classically chaotic, then $U^{n}$ is more chaotic, at least in the sense

of a higher rate of phase-space mixing. But we have seen here that higher powers of $U$ may be less entangling [39]. 


\section{Acknowledgments}

We thank O. Bohigas, A. M. Ozorio de Almeida, M. Sieber, F. Toscano, C. Viviescas, and

K. Zyczkowski, for interesting comments. Partial financial support from CNPq, CAPES, PROSUL, and The Millennium Institute for Quantum Information (Brazilian agencies) is gratefully acknowledged.

[1] K. Furuya, M. C. Nemes, and G. Q. Pellegrino, Phys. Rev. Lett. 80, 5224 (1998).

[2] T. Gorin and T. H. Seligman, J. Opt. B: Quantum Semiclass. Opt. 4, S386 (2002); Phys. Lett. A 309, 61 (2003).

[3] Ph. Jacquod, Phys. Rev. Lett. 92, 150403 (2004).

[4] M. Znidaric and T. Prosen, Phys. Rev. A 71, 032103 (2005).

[5] R. M. Angelo and K. Furuya, Phys. Rev. A 71, 042321 (2005).

[6] H. Kubotani, M. Toda, and S. Adachi, Phys. Rev. A 74, 032314 (2006).

[7] C. Petitjean and Ph. Jacquod, Phys. Rev. Lett. 97, 194103 (2006).

[8] C. Pineda and T. H. Seligman, Phys. Rev. A 75, 012106 (2007).

[9] P. A. Miller and S. Sarkar, Nonlinearity 12, 419 (1998).

[10] P. A. Miller and S. Sarkar, Phys. Rev. E 60, 1542 (1999).

[11] A. Lakshminarayan, Phys. Rev. Lett. 64, 036207 (2001).

[12] J. N. Bandyopadhyay and A. Lakshminarayan, Phys. Rev. Lett. 89, 060402 (2002).

[13] A. Tanaka, H. Fujisaki, and T. Miyadera, Phys. Rev. E. 66, 045201(R) (2002); 67, 066201 (2003).

[14] A. J. Scott and C. M. Caves, J. Phys. A 36, 9553 (2003).

[15] R. Demkowicz-Dobrzanski and M. Kus, Phys. Rev. E 70, 066216 (2004).

[16] J. N. Bandyopadhyay and A. Lakshminarayan, Phys. Rev. E 69, 016201 (2004).

[17] S. Ghose and B. C. Sanders, Phys. Rev. A 70, 062315 (2004).

[18] D. Rossini, G. Benenti, and G. Casati, Phys. Rev. E 74, 036209 (2006).

[19] M. Pogorzelska and R. Alicki, arXiv:quant-ph/0611092 (2006).

[20] P. Zanardi, C. Zalka, and L. Faoro, Phys. Rev. A 62, 030301 (2000).

[21] O. Bohigas, M. J. Giannoni, and C. Schmit, Phys. Rev. Lett. 52, 1 (1984). 
[22] F. Haake, Quantum Signatures of Chaos (Springer-Verlag, Berlin, 2000).

[23] R. O. Vallejos, P. R. del Santoro, and A. M. Ozorio de Almeida, J. Phys. A 39, 5163 (2006).

[24] R. F. Abreu and R. O. Vallejos, Phys. Rev. A 73, 052327 (2006).

[25] P. Zanardi, Phys. Rev. A 63, 040304 (2001).

[26] X. Wang and P. Zanardi, Phys. Rev. A 66, 044303 (2002).

[27] M. A. Nielsen et al, Phys. Rev. A 67, 052301 (2003).

[28] M. Nielsen and I. Chuang, Quantum Computation and Quantum Information (Cambridge University Press, Cambridge, 2001).

[29] W. K. Wootters, Foundations of Physics 20, 1365 (1990).

[30] I. Bengtsson and K. Zyczkowski, Geometry of Quantum States - An Introduction to Quantum Entanglement (Cambridge University Press, Cambridge, 2006).

[31] M. L. Mehta, Random Matrices (Academic Press, New York, 2004).

[32] K. Zyczkowski (private communication).

[33] J. N. Bandyopadhyay and A. Lakshminarayan, arXiv:quant-ph/0504052.

[34] R. F. Abreu, PhD Dissertation (in preparation).

[35] S. Aubert and C. S. Lam, J. Math. Phys. 44, 6112 (2003).

[36] D. Braun, J. Phys. A 39, 14581 (2006).

[37] T. Gorin, J. Math. Phys. 43, 3342 (2002).

[38] D. A. Meyer and N. R. Wallach, J. Math. Phys. 43, 4273 (2002); G. K. Brennen, Quantum Inf. Comput. 3, 619 (2003).

[39] Compare with T. Prosen and T. H. Seligman, J. Phys. A 35, 4707 (2002). 